\title{
Study on Electromagnetic Radiation Interference Caused by Rocket Fuel
}

\section{Yuanbo Cui}

Nanjing University of Science and Technology

Deren Kong ( $\nabla$ derenkong@126.com )

Nanjing University of Science and Technology

Jian Jiang

Nanjing University of Science and Technology

Shang Gao

Nanjing University of Science and Technology

Shuai Wang

National University of Defense Technology

\section{Research Article}

Keywords: Electromagnetic Radiation, Fuel

Posted Date: October 21st, 2021

DOl: https://doi.org/10.21203/rs.3.rs-965825/v1

License: (c) (1) This work is licensed under a Creative Commons Attribution 4.0 International License. Read Full License

Version of Record: A version of this preprint was published at Sensors on December 4th, 2021. See the published version at https://doi.org/10.3390/s21238123. 


\title{
Study on Electromagnetic Radiation Interference Caused by Rocket Fuel
}

\author{
Yuanbo Cui ${ }^{1}$, Deren Kong ${ }^{1 *}$, Jian Jiang ${ }^{1}$, Shang Gao', Shuai Wang² \\ ${ }^{1}$ School of Mechanical Engineering, Nanjing University of Science and Technology, Nanjing, 210094, China \\ ${ }^{2}$ College of Intelligent Science and Technology, National University of Defense Technology, Changsha, 410072 , \\ China \\ "corresponding.derenkong@126.com
}

\begin{abstract}
During the launch and return of a spacecraft, the intense combustion of propellants generates strong electromagnetic radiation, which interferes with the operation of electronic equipment in the spacecraft. To improve the electromagnetic compatibility of electronic equipment in spacecraft, it is necessary to study the electromagnetic radiation characteristics of rocket fuel. An electromagnetic radiation measurement system based on antennas is designed to measure the electromagnetic radiation generated by rocket fuel, and the electromagnetic radiation characteristics are obtained through data analysis. The mechanism of the electromagnetic radiation generated by rocket fuel is comprehensively analysed through the spatial, time-domain, frequency-domain, and energy-domain characteristics. A characterization model is established to provide a reliable scheme for evaluating the influence of rocket fuel electromagnetic radiation on electronic equipment in spacecraft.
\end{abstract}

\section{Introduction}

On May 30, 2020, SpaceX successfully launched the Falcon 9 Rocket "Crew Dragon" from the Kennedy Space Center in Florida, and two NASA astronauts were sent into space, which is the first time in human history that a commercial company completed a manned spaceflight mission. A very important step during the launch process is the first-stage rocket recovery process, as shown in Figure 1. The task of first-stage rocket recovery was carried out at sea. During the live broadcast, it was noticed that the broadcast was interrupted for a few seconds when the rocket approached the recovery ship and was about to land. In fact, most live broadcasts of first-stage rocket recoveries are temporarily interrupted during rocket landings at sea. While a dedicated video cable is used to transmit video signals during rocket recoveries on land, video signals are transmitted to broadcast rooms using satellite communications during rocket recoveries at sea. When a rocket approaches a recovery ship at sea, the rocket fuel burns to produce intense electromagnetic radiation, which causes electromagnetic interference with wireless communication so that live broadcast signal interruption always occurs during the process of first-stage rocket recovery at sea. Energetic materials combustion can produce electromagnetic radiation that is enough to cause the malfunction of electronic equipment in spacecraft ${ }^{1,2}$. Affecting the live video signal is not enough to have a large adverse effect, but the heat generated by violent combustion can cause the plasma state around the spacecraft to change so that the electromagnetic radiation generated by deflagration can adversely affect electronic equipment in spacecraft, especially communication equipment ${ }^{3}$. 


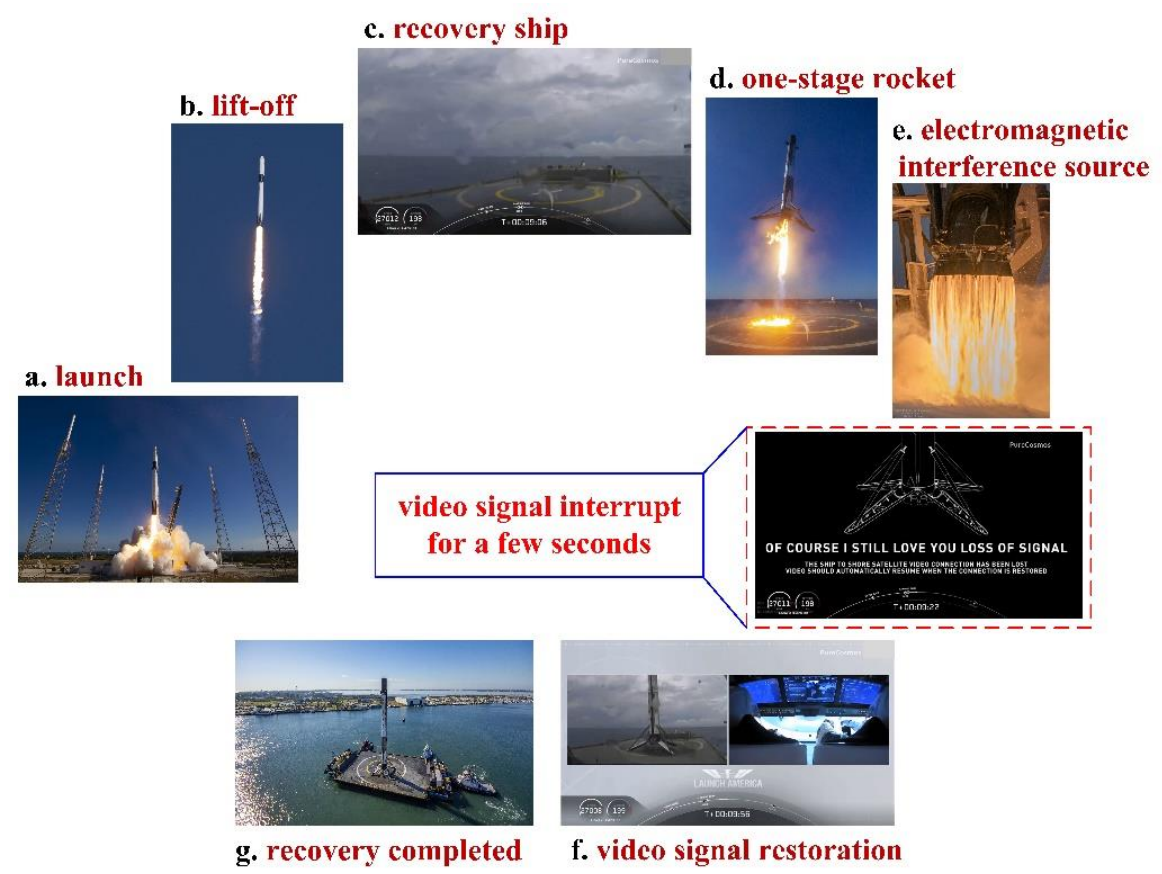

Figure 1. Recovery process of the first-stage rocket of a Falcon 9

China launched the Shenzhou XII spacecraft from the Jiuquan Satellite Launch Center on June 17, 2021, its seventh manned spaceflight, sending three astronauts to the core module of Tianhe Space Station. After completing a three-month work task, the Shenzhou XII spacecraft returned to earth and successfully landed at the Dongfeng Landing Site on September 17, 2021. Figure 2 shows that when spacecraft return to the earth, due to the excessive speed, the surface of the spacecraft and the surrounding gas friction generate substantial huge heat, forming a high-temperature plasma gas layer on the surface of the spacecraft, which blocks electromagnetic waves and causes the spacecraft lose contact with the ground ${ }^{4}$.

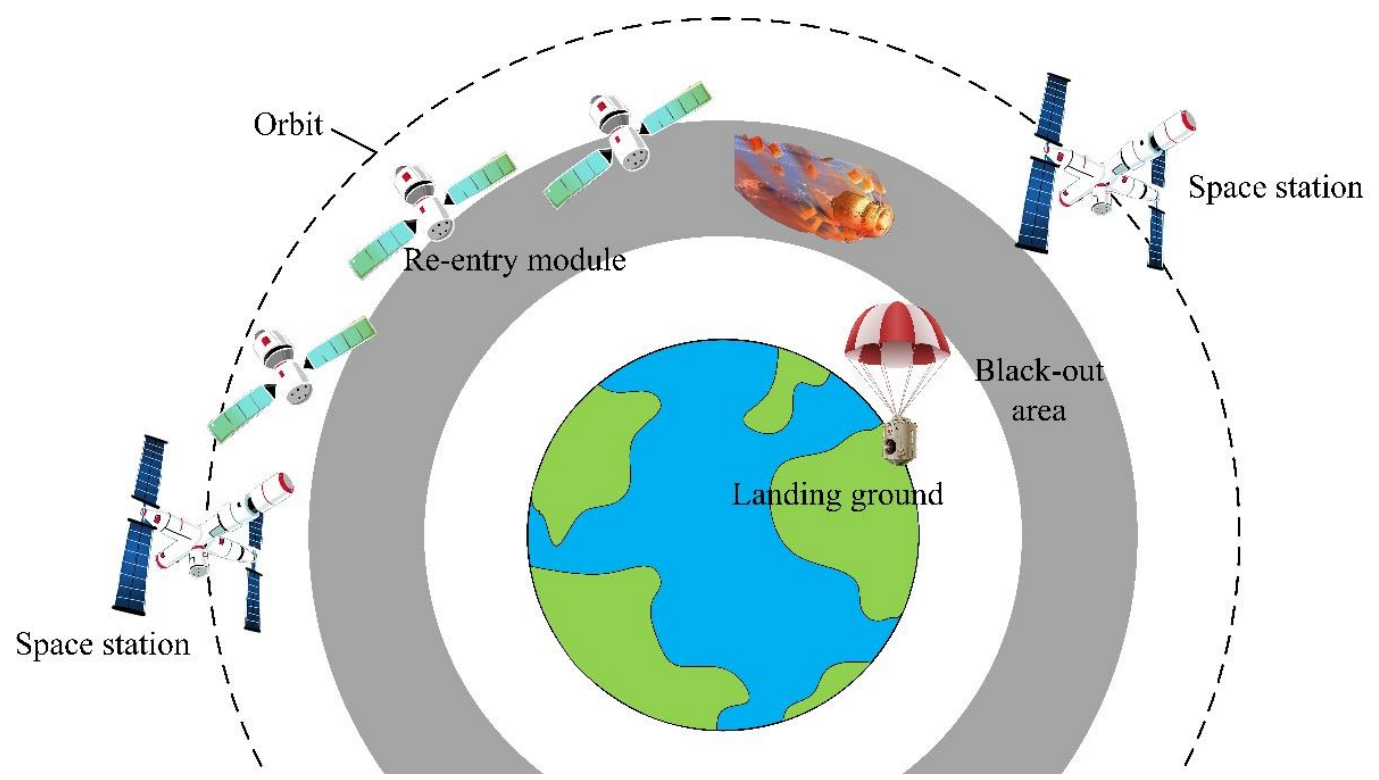

Figure 2. The return process of the Shenzhou XII spacecraft

In 1954, Kolsky first discovered the phenomenon in which electric pulses appeared during the explosion of energetic materials ${ }^{5}$. In the following decades, related studies on electromagnetic radiation produced by explosions of energetic materials were carried out one after another. However, due to the high cost, difficulty and reproducibility of experiments, the progress of related studies has been slow. In 1982, van Lint VAJ used broadband low-frequency, narrowband VHF and ultrahigh frequency sensitive sensors to measure the electromagnetic radiation generated by fuel deflagration ${ }^{6,7}$. The experimental results proved that the fireball and air impact areas around fuel deflagration contained a large radial electric 
field. The frequency of the electromagnetic signal observed in the experiment was mainly concentrated at $57 \mathrm{MHz}$, and the average electric field was $10^{5} \mathrm{~V} / \mathrm{m}$. In 1990, Boronin studied the physical mechanism of an electromagnetic field generated by the deflagration of condensed fuel ${ }^{8}$. Boronin suggested that at the initial moment of metal deformation and failure, potential energy in both gaseous and solid-state flowed out from cracks, then electrokinesis and potential energy friction resulted in the electrical polarity of the gaseous and solid shells being opposite, and the space charge of the gas and solid potential energy formed effective dipoles because of the asymmetrical scattering of the potential energy. The viewpoint that the mechanism of generating radioradiation through deflagration is related to the acceleration or deceleration of certain electron genes in the ionized air layer at the front of a shock wave is called "Boronin Effect" . In 2011, Cao Jingyang used pole antennas and real-time spectrometers to measure the electromagnetic radiation caused by the deflagration of aerospace explosives. Cao found that the electromagnetic radiation generated by the deflagration of shaped fuel had the multi-pulse and broadband characteristics, electronic pulse duration of about tens of microseconds, and frequency distributed below megahertz. It was also found that the energy of the electromagnetic radiation was positively correlated with the quality of fuel, and the electromagnetic pulse was delayed for hundreds of microseconds and continued for a long time after combustion ended ${ }^{10-12}$. In 2014, A.L.Kuhl summarized and analysed the experimental results of electromagnetic radiation generated during energetic material deflagration. It was believed that the movement of ionized atoms, ions and electrons was the cause of the electromagnetic waves caused by deflagration. The expansion of detonation products caused a strong vibration in the surrounding air, forming a strong thermal wave (T 11000 K) with a duration of $20 \mu \mathrm{s}$, which caused clear ionization in the air and movement of ion plaques generating a current ${ }^{13}$.

\section{Methods}

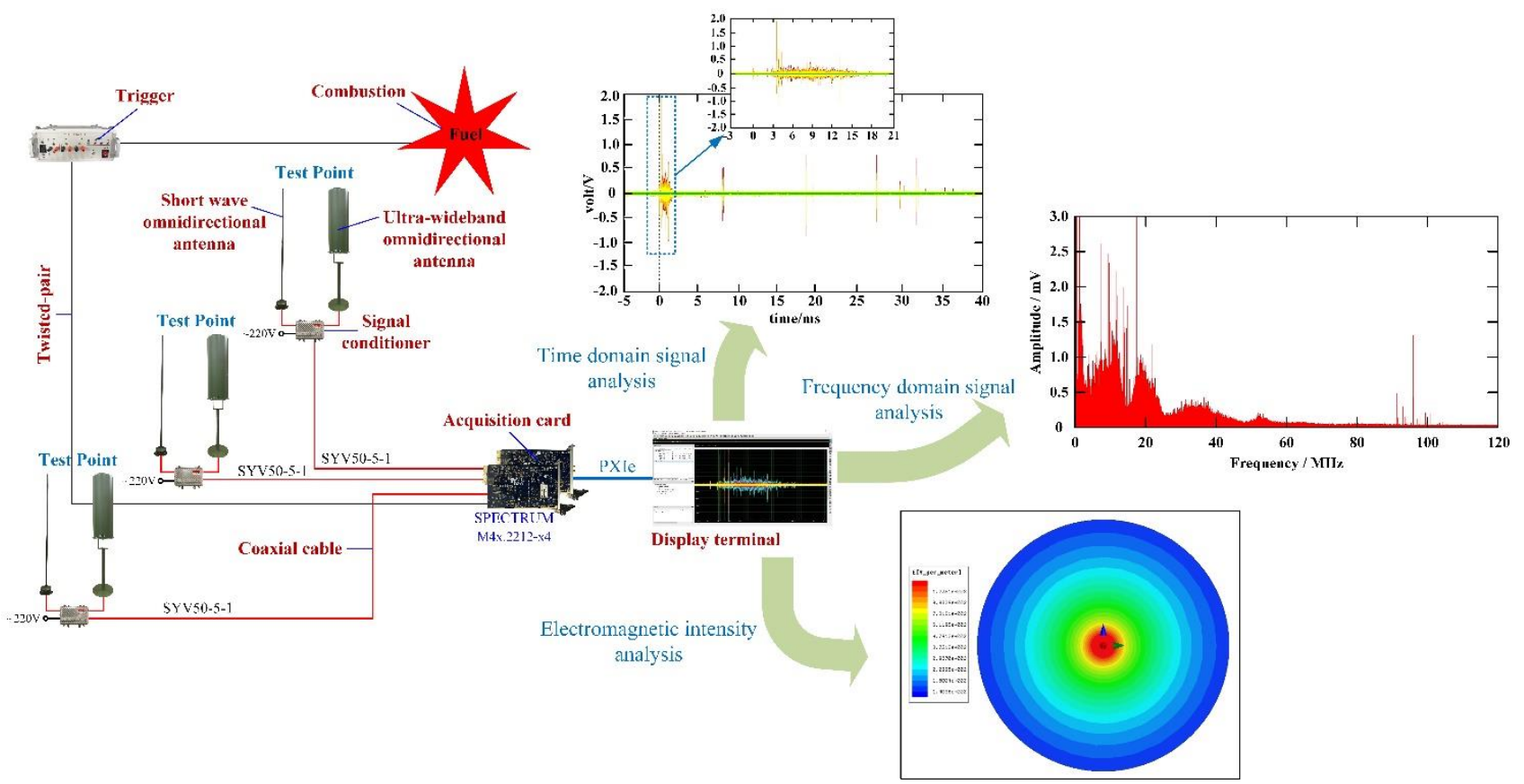

Figure 3. Electromagnetic radiation measurement and analysis process

Figure 3 shows that an electromagnetic radiation measurement system based on an ultrawideband omnidirectional antenna and a shortwave omnidirectional antenna is designed to measure the characteristics of electromagnetic radiation generated by rocket fuel ${ }^{14}$. The time-domain characteristics, spectrum distribution and intensity of electromagnetic radiation are analysed as the experiment ends. In this paper, a shortwave antenna and ultrawideband antenna are cooperatively adopted to measure electromagnetic radiation, covering the frequency band up to $3 \mathrm{GHz}$, and a high-speed acquisition card is used to record data with time-domain signal accuracy of being $10^{-9} \mathrm{~s}$ and a recording time as long as $1000 \mathrm{~ms}$. As shown in Figure 3, the test point consists of a shortwave passive omnidirectional antenna, an ultrawideband passive omnidirectional antenna and a signal conditioner. The sampling bandwidth of the shortwave passive omnidirectional antenna is 1.5 $\mathrm{MHz} 30 \mathrm{MHz}$, which adopts a vertical polarization mode with a standing-wave ratio (SWR) less than 2.5 and a gain greater than $-35 \mathrm{dBi}$. The ultrawideband omnidirectional antenna uses a double-cone loading structure with a vertical 
polarization method, the sampling bandwidth is $30 \mathrm{MHz} 3 \mathrm{GHz}$, the gain is greater than $-15 \mathrm{dBi}$, and the output impedance of both the shortwave antenna and ultrawideband antenna is $50 \Omega$. The signal conditioner possesses multiple functions, including a combiner, signal amplifier and limiter, which can combine two electromagnetic signals of different frequencies and amplify the signal at the same time with a range of amplification factors of $10 \sim 30 \mathrm{~dB}$. The function of the limiter with limiting power greater than $10 \mathrm{~W}$ is to prevent the signal power from being excessively high and damaging acquisition card $^{15-18}$.

Additionally, what can be clearly seen in Figure 3 is the process of analyzing the electromagnetic signal. In the sampling period, the delay time, peak arrival time and duration time of the electromagnetic signal are important parameters of the time-domain analysis. Through the Sbench data processing program, various parameters of an electromagnetic signal can be obtained very accurately. The frequency spectrum distribution of an electromagnetic signal can be obtained by performing a fast Fourier transform on the time-domain signal of the electromagnetic radiation, but a simple fast Fourier transform can cover sampling points with smaller amplitudes. Since the electromagnetic radiation generated by fuel deflagration is a random unknown signal, using a Fourier transform with a Hanning window can extract the maximum effective electromagnetic spectrum. Electromagnetic radiation intensity is the most important data for preventing electromagnetic hazards. Since the fuel used in aerospace missions is of very high mass and has a strong deflagration energy, it is particularly necessary to consider the electromagnetic wave propagation loss when analysing the electromagnetic field intensity ${ }^{19,20}$.

\section{Electromagnetic radiation modelling}

Electromagnetic radiation propagates from a radiation source to space through a certain medium in the form of electromagnetic waves, while the mechanism of electromagnetic radiation around a spacecraft is embodied in the influence and threat of the radiation ${ }^{21}$. The electromagnetic environment around a spacecraft is a comprehensive reflection of the complex distribution and changes in the electromagnetic radiation generated by aerospace fuel in terms of space, time, frequency spectrum and power, which is a direct way to describe the external characteristics of the electromagnetic environment around a spacecraft as its external appearance ${ }^{22}$. This paper analyses and models the electromagnetic environment around a spacecraft through four aspects, the spatial characteristics, time-domain characteristics, frequencydomain characteristics and energy-domain characteristics, to superiorly evaluate the influence and threat of electromagnetic radiation on electronic equipment in spacecraft. Each electronic device in a spacecraft can be regarded as an electromagnetic sensor, and the electromagnetic interference on every electromagnetic sensor is affected by many parameters, such as the operating time, induction direction, working frequency, working bandwidth, separation distance and frequency offset ${ }^{23}$. As shown in Figure 4(A), these parameters have different effects on the electromagnetic compatibility of the electronic equipment; additionally, the exterior parameters have priority over the interior parameters when calculating electromagnetic interference. That is the exterior parameters are more important in the process of electromagnetic interference calculation.

The time-varying power density spectrum $\mathrm{S}(\boldsymbol{r}, t, f)$ is a complicated mathematical expression, which cannot describe the electromagnetic environment simply and intuitively. How to use several indicators to describe the electromagnetic radiation characteristics caused by rocket fuel and build an electromagnetic environment model, which is a question worth exploring. As shown in Figure 4(B), in order to evaluate the electromagnetic environment caused by rocket fuel, in article it uses three indicators of space coverage, time occupancy and frequency occupancy to describe the characteristics of electromagnetic radiation. The expression of space coverage is as shown in Equation 1, the expression of time occupancy is as shown in Equation 2, the expression of frequency occupancy is as shown in Equation 3. The meaning of the parameters in the equations is shown in Table 1.

$$
\begin{aligned}
& \mathrm{SO}=\frac{1}{V_{\Omega}} \int_{\Omega} U\left[\frac{1}{f_{2}-f_{1}} \frac{1}{t_{2}-t_{1}} \int_{t 1}^{t_{2}} \int_{f_{1}}^{f_{2}} S(\boldsymbol{r}, t, f) d f d t-S_{0}\right] d \tau \\
& \mathrm{TO}=\frac{1}{t_{2}-t_{1}} \int_{t_{1}}^{t_{2}} U\left[\frac{1}{\left(f_{2}-f_{1}\right) V_{\Omega}} \int_{\Omega} \int_{f_{1}}^{f_{2}} S(\boldsymbol{r}, t, f) d f d \tau-S_{0}\right] d t
\end{aligned}
$$




$$
\mathrm{FO}=\frac{1}{f_{2}-f_{1}} \int_{f_{1}}^{f_{2}} U\left[\frac{1}{\left(t_{2}-t_{1}\right) V_{\Omega}} \int_{\Omega} \int_{t_{1}}^{t_{2}} S(\boldsymbol{r}, t, f) d t d \tau-S_{0}\right] d f
$$

\begin{tabular}{|l|l|l|}
\hline Parameter & Explain & Unit \\
\hline$\theta_{1}$ & Start angle of the scanning azimuth of Device A & o \\
\hline$\theta_{2}$ & End angle of the scanning azimuth of Device A & o \\
\hline$\varphi_{1}$ & Start angle of the pitch scanning azimuth of Device & o \\
\hline$\varphi_{2}$ & End angle of the pitch scanning azimuth of Device A & o \\
\hline $\mathrm{U}$ & Unit step function & - \\
\hline$f_{1}$ & Start frequency of Device A & $\mathrm{MHz}$ \\
\hline$f_{2}$ & Stop frequency of Device A & $\mathrm{MHz}$ \\
\hline$t_{1}$ & Start time of Device A & $\mathrm{s}$ \\
\hline$t_{2}$ & End time of Device A & $\mathrm{s}$ \\
\hline $\mathrm{S}[\theta(t), \varphi(t), f]$ & $\begin{array}{l}\text { Average power density spectrum of the electromagnetic } \\
\text { environment around Device A }\end{array}$ & $\mathrm{W} /\left(\mathrm{m}^{2} \cdot \mathrm{Hz}\right)$ \\
\hline$\theta(t)$ & Azimuth angle of Device X relative to Device A at time t & $\mathrm{o}$ \\
\hline$\varphi(t)$ & Pitch angle of Device X relative to Device A at time t & $\mathrm{o}$ \\
\hline$S_{0}$ & $\begin{array}{l}\text { Electromagnetic environment threshold for normal } \\
\text { operation of Device A }\end{array}$ & $\mathrm{W} /\left(\mathrm{m}^{2} \cdot \mathrm{Hz}\right)$ \\
\hline$f_{\text {rmax }}$ & Upper limit of the working bandwidth of Device A & $\mathrm{MHz}$ \\
\hline$f_{r \min }$ & Lower limit of the working bandwidth of Device A & $\mathrm{MHz}$ \\
\hline$f_{\text {jmax }}$ & Upper limit of the signal bandwidth of the radiation source & $\mathrm{MHz}$ \\
\hline$f_{\text {jmin }}$ & Lower limit of the signal bandwidth of the radiation source & $\mathrm{MHz}$ \\
\hline
\end{tabular}

Table 1. Parameter meaning

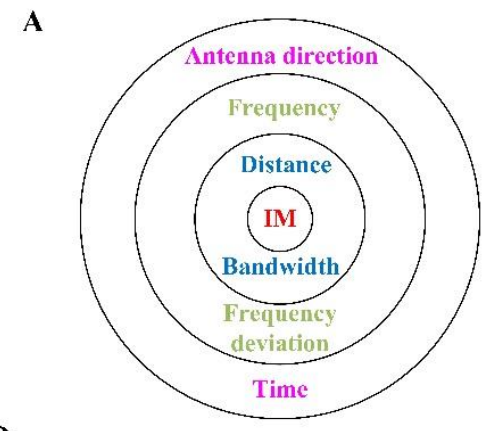

D

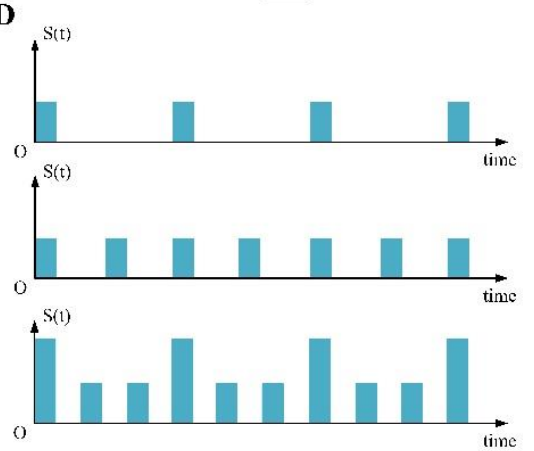

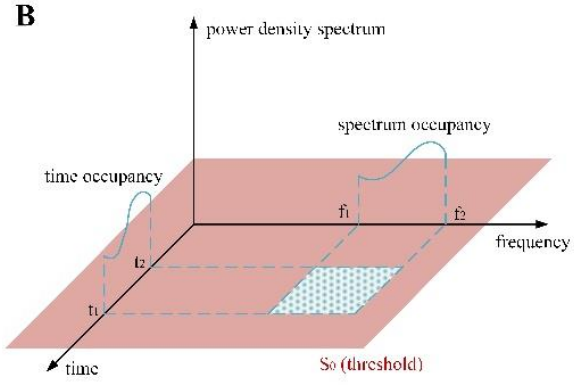

$$
\mathbf{E}
$$
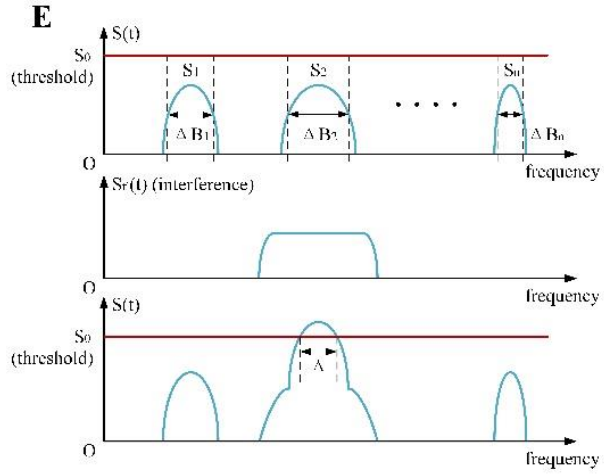

C

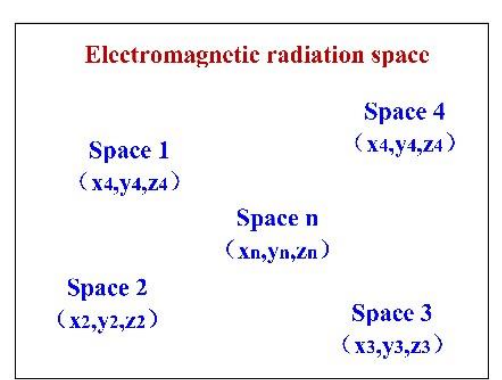

F
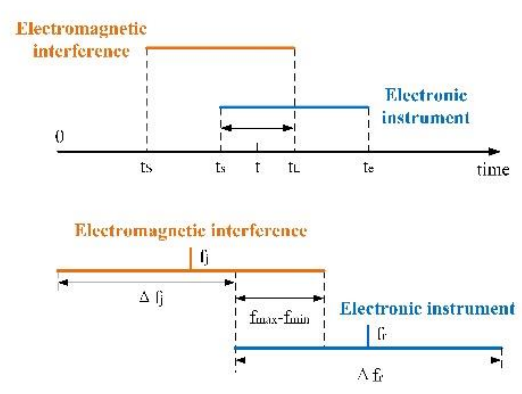

Figure 4. Electromagnetic environment analysis and modelling 


\section{Analysis and modelling of the spatial characteristics of the electromagnetic environment}

The spatial characteristics represent the distribution of electromagnetic radiation in different spaces and the changes of the electromagnetic signals with space, which is strictly represented by an electromagnetic signal power density spectrum corresponding to a specific location. According to the theory of electromagnetics, the electromagnetic environment at any point in space can be represented by an electric field intensity $\mathbf{E}(\mathbf{r}, \mathrm{t})$, where $\mathbf{r}$ represents the three-dimensional coordinates of some point in space and t represents time. For plane electromagnetic waves, the power density of an electromagnetic signal at a certain position in space is proportional to $\mathbf{F}(\mathbf{r}, \mathrm{t}) \times \mathbf{F}^{*}(\mathbf{r}, \mathrm{t})$. Through a Fourier transform, $\mathbf{S}(\mathbf{r}, \mathrm{t}$, f), the time-varying power density spectrum of the time-varying correlation function of analytic signal $\mathbf{F}(\mathbf{r}, t)$ of $\mathbf{E}(\mathbf{r}, t)$ can be obtained. $\mathbf{S}(\mathbf{r}, \mathbf{t}, \mathrm{f})$ expresses the electromagnetic energy that flows through a unit area and a unit bandwidth at a certain spatial location at any time with any frequency. The signal intensity of the electromagnetic wave generated during deflagration at any position can be expressed by the power density spectrum $\mathbf{S}(\mathbf{r}, \mathbf{t}, \mathbf{f})$ after propagating through some medium $^{24,25}$.

During the launch or return of spacecraft, the electromagnetic radiation generated by combustion of rocket fuel does not present a point-like distribution but a three-dimensional state. For the electronic equipment on spacecraft, there are multiple radiation sources, as shown in Figure 4(C), which assums that there are many points in the radiation area with one radiation source, and their three-dimensional coordinates are expressed as $\mathbf{r}_{\mathrm{i}}(\mathrm{i}=1 \sim \mathrm{n})$. At a certain time, each radiation source radiates a signal with a certain pattern and frequency, the electric field intensity of which is $\mathbf{E}_{\mathrm{i}}\left(\mathbf{r}_{\mathrm{i}}, \mathrm{t}\right)$ and the power density spectrum is $\mathbf{S}_{i}\left(\mathbf{r}_{i}, t, f\right)$, which is an electromagnetic signal whose intensity and frequency change with time. Since the intensity of electromagnetic radiation generated by different radiation sources is different and its direction changes with time, the electromagnetic radiation on electronic equipment generated by each source needs to be multiplied by a vector factor $\mathbf{A}_{\mathrm{i}}$ that varies with space and time, which is related to the propagation path. Therefore, the electromagnetic field intensity of radiation from $\mathbf{r}_{i}$ to $\mathbf{r}_{\mathrm{j}}$ is $\mathbf{A}_{\mathrm{i}} \mathbf{E}_{\mathrm{i}}\left(\mathbf{r}_{\mathrm{ji}}, \mathrm{t}-\mathrm{t}_{\mathrm{ij}}\right)$. The electromagnetic radiation received by point $\mathrm{j}$ can be expressed as the power spectral density $\mathbf{S}_{\mathbf{j}}\left(\mathbf{r}_{\mathbf{j}}, \mathrm{t}, \mathrm{f}\right)$ of the combined intensity in Equation 4 . Without loss of generality, $\mathbf{S}_{\mathrm{j}}\left(\mathbf{r}_{\mathrm{j}}, \mathrm{t}, \mathrm{f}\right)$ can be written as $\mathbf{S}(\mathbf{r}, \mathrm{t}, \mathrm{f})$, which is the intensity of electromagnetic radiation at any spatial point in the presence of $\mathrm{n}$ radiation sources.

$$
E_{j}\left(r_{j}, t\right)=\sum_{i=1 \sim m} A_{i} E_{i}\left(r_{i j}, t-t_{i j}\right)
$$

According to Equation 4, within a certain time range $\left[\mathrm{t}_{1}, \mathrm{t}_{2}\right]$ and frequency range $\left[\mathrm{f}_{1}, \mathrm{f}_{2}\right]$, the signal intensity of any point $\mathrm{r}$ can be expressed using Equation 5 by the average power density spectrum, where the double integral is respectively the integration over time and frequency.

$$
\mathrm{S}(\mathbf{r})=\frac{1}{\left(t_{2}-t_{1}\right)\left(f_{2}-f_{1}\right)} \int_{t_{1}}^{t_{2}} \int_{f_{1}}^{f_{2}} S(\boldsymbol{r}, t, f) d f d t
$$

Spatial threat assessment modelling uses a spatial threat level as an indicator to quantitatively analyse the threat of an electromagnetic environment with electronic equipment as a threatened object. The spatial threat degree of the electromagnetic environment to the electronic equipment is expressed by the ratio of the azimuth and elevation range where the average power density spectrum of the electromagnetic environment exceeds the electromagnetic environment threshold to the working azimuth and elevation range of Device A. According to Equation 1, the spatial threat degree of Device A is:

$$
\mathrm{ST}=\frac{1}{\theta_{2}-\theta_{1}} \cdot \frac{1}{\varphi_{2}-\varphi_{1}} \int_{\varphi_{1}}^{\varphi_{2}} \int_{\theta_{1}}^{\theta_{2}} U\left\{\frac{1}{f_{2}-f_{1}} \cdot \frac{1}{t_{2}-t_{1}} \int_{f_{1}}^{f_{2}} \int_{t_{1}}^{t_{2}} S[\theta(t), \varphi(t), f] d t d f-S_{0}\right\} d \theta d \varphi
$$

\section{Analysis and modelling of the time-domain characteristics of the electromagnetic environment}

The time-domain characteristic represents the change in the electromagnetic radiation signal over time, which is expressed as the distribution of the electromagnetic radiation signal over a time series, which can usually be expressed by parameters such as the signal density exceeding a certain intensity per unit time. The electromagnetic radiation produced by rocket fuel combustion has both pulse radiation and continuous radiation, whose distribution is different in different periods and has dynamic variability. Pulse signal density is usually expressed by the number of pulses per unit time, and continuous signal density is usually expressed by the number of different signals per unit time ${ }^{26}$. In Equation 4 , the signal 
power density spectrum at any position $\mathbf{r}_{\mathrm{j}}$ in space can be expressed as $S\left(\mathbf{r}_{\mathrm{j}}, \mathrm{t}, \mathrm{f}\right)$. When the location, number, propagation path and antenna gain of an explosive radiation source are determined, the strength of the signal at the measured point, that is the power density of the signal, is only a function of time. In a certain explosion space $V_{\Omega}$ and frequency range $\left[f_{1}\right.$, $\mathrm{f}_{2}$ ], the change law of signal intensity with time can be expressed by average power density as Equation 7, where the double integration is the integration over frequency and space.

$$
\mathrm{S}(\mathrm{t})=\frac{1}{V_{\Omega}\left(f_{2}-f_{1}\right)} \int_{\Omega} \int_{f_{1}}^{f_{2}} S(\boldsymbol{r}, t, f) d f d \Omega
$$

What can be clearly seen in Figure 4(D) is that $\mathrm{S}(\mathrm{t})$ represents the signal power density in a specific space and within a certain frequency band, and a larger value of $\mathrm{S}(\mathrm{t})$ represents the a more complex electromagnetic environment. For the first and second coordinate axes in Figure 4(D), the vertical axis denotes pulse intensity, the horizontal axis denotes time, and the third coordinate axis represents the overall signal intensity of $\mathbf{r}_{\mathrm{j}}$, which shows that the intensity and density of electromagnetic radiation are different at different times.

The time-domain correlation has only four states: the operating or nonoperating of Device A and the generation and disappearance of a radiation source at time t. Electromagnetic radiation can affect Device A only when Device A is working at time $t$ and a radiation is being generated. Assuming that the start time of electromagnetic interference is $t_{S}$, the end time is $t_{E}$, the operating start time of Device $A$ is $t_{s}$, and the operating end time of Device $A$ is $t_{e}$, as shown in Figure 4(F), according to the operating period of Device A and the interference period of the electromagnetic radiation, the timedomain correlation calculation model at time $t$ is as follows:

$$
\mathrm{TR}=\left\{\begin{array}{cl}
1, & t \in\left[t_{s}, t_{E}\right] \cap\left[t_{s}, t_{e}\right] \\
0, & \text { others }
\end{array}\right.
$$

Time-domain threat assessment modelling uses a threat degree as an indicator to quantitatively analyse the time-domain threat of the electromagnetic environment with equipment as the threatened object. The time-domain threat degree of an electromagnetic environment to Device A is expressed by the ratio between the period when the average power density spectrum of the electromagnetic environment threshold to the working period of Device A. According to Equation 2, the time-domain threat degree of Device A is:

$$
\mathrm{TT}=\frac{1}{t_{2}-t_{1}} \int_{t_{1}}^{t_{2}} U\left\{\frac{1}{\theta_{2}-\theta_{1}} \cdot \frac{1}{\varphi_{2}-\varphi_{1}} \cdot \frac{1}{f_{2}-f_{1}} \int_{\theta_{1}}^{\theta_{2}} \int_{\varphi_{1}}^{\varphi_{2}} \int_{f_{1}}^{f_{2}} S[\theta(t), \varphi(t), f] d f d \varphi d \theta-S_{0}\right\} \mathrm{dt}
$$

\section{Analysis and modelling of the frequency-domain characteristics of the electromagnetic environment}

The frequency-domain characteristics represent the overall state of the spectrum occupied by the spacecraft's electromagnetic environment, usually expressed by parameters such as frequency occupancy. Frequency occupancy is the ratio of the frequency band occupied by the signal power density spectrum of the electromagnetic environment in a certain space and time interval that exceeds the specified environmental level threshold to the frequency range of Device A, which reflects the situation of how much of the electromagnetic spectrum resources are occupied by the space's electromagnetic radiation ${ }^{27,28}$. According to Equation 4 , in a certain space of $\mathrm{V}_{\Omega}$ and time range of $\left[\mathrm{t}_{1}, \mathrm{t}_{2}\right]$, the average power spectrum of the signal at different frequencies can be expressed using Equation 10, where the double integration is used to integrate over time and space.

$$
\mathrm{S}(f)=\frac{1}{V_{\Omega}\left(t_{2}-t_{1}\right)} \int_{\Omega} \int_{t_{1}}^{t_{2}} S(\boldsymbol{r}, t, f) d t d \Omega
$$

According to the calculation method of the spectrum range, the spectrum range occupied by the electromagnetic radiation signal in a certain time and space can be calculated from the average power spectrum function of the signal strength represented by Equation 10, which is abbreviated as $\Delta \mathrm{B}$. For the electronic equipment of spacecraft, it is assumed that there are different radiation frequencies of $\mathrm{f}_{\mathrm{k}}(\mathrm{k}=1 \sim \mathrm{n})$ due to the joint action of $\mathrm{n}$ radiation sources, and each radiation source has a different spectrum range of $\Delta \mathrm{B}_{\mathrm{k}}(\mathrm{k}=1 \sim \mathrm{m})$. If there is no overlapping part of the spectrum, that is, there is no self-interference or mutual interference, as shown in the first coordinate axis of Figure 4(E), then the spectrum occupancy is defined as 0 , which is $\Delta \mathrm{B}=\sum_{k=1 \sim n} \Delta B_{k}$. However, in actual situations there will be interference from other signals in the range of $\Delta \mathrm{B}$, and frequency overlap will occur; then, $\mathrm{S}(f)$ must exceed the allowable threshold $S_{0}$. As shown in the 
second and third coordinate axes of Figure 4(E), the spectrum occupancy will not be 0 , if the spectrum width exceeding the threshold is $\Delta$, the spectrum occupancy is $\mathrm{FO}=\frac{\Delta}{\Delta \mathrm{B}}$, and the electromagnetic environment becomes more complicated with the spectrum occupancy being greater.

It quantitatively describes the relevant conditions and calculates the degree of correlation in the frequency domain: assuming that bandwidth of the linear part of Device A is $\Delta f_{r}$, the central frequency of Device A is $f_{r}$, the signal bandwidth of the radiation source is $\Delta f_{j}$, the central frequency of the signal is $f_{j}$, clearly when the power and bandwidth of the signal or receiver are determined, the signal power of a jamming device is related to the frequency aiming error of $\delta_{f}$ and $\frac{\Delta f_{j}}{\Delta f_{r}}$. Due to the frequency aiming error of $\delta_{f}$, the central frequency of the radiation source cannot be completely aligned with the central frequency of Device A, and the bandwidth of the radiation source cannot completely cover the working bandwidth of Device A, so the degree of coincidence between the signal bandwidth of the radiating source and of receiver is used to determine the frequency-domain correlation, as shown in the second coordinate axis in Figure 4(F).

The frequency-domain correlated calculation model is as follows:

$$
\left\{\begin{array}{c}
\mathrm{FR}=\frac{f_{\text {max }}-f_{\text {min }}}{\Delta f_{r}} U\left(f_{\text {max }}-f_{\text {min }}\right) \\
\Delta f_{r}=f_{\text {max }}-f_{\text {min }} \\
f_{\text {max }}=\min \left[f_{r_{\text {max }}}, f_{\text {jmax }}\right], f_{\text {min }}=\max \left[f_{\text {rmin }}, f_{\text {jmin }}\right]
\end{array}\right.
$$

Frequency-domain threat assessment modelling takes a frequency-domain threat degree as an indicator to quantitatively analyse the frequency-domain threat generated by the electromagnetic environment. The frequency-domain threat of the electromagnetic environment to Device A is expressed by the frequency band where the average power density spectrum of the electromagnetic radiation exceeds the electromagnetic environment threshold and the working frequency band of Device A. According to Equation 3, the frequency-domain threat of Device A is as follows:

$$
\mathrm{FT}=\frac{1}{f_{2}-f_{1}} \int_{f_{1}}^{f_{2}} U\left\{\frac{1}{\theta_{2}-\theta_{1}} \cdot \frac{1}{\varphi_{2}-\varphi_{1}} \cdot \frac{1}{t_{2}-t_{1}} \int_{\theta_{1}}^{\theta_{2}} \int_{\varphi_{1}}^{\varphi_{2}} \int_{t_{1}}^{t_{2}} S[\theta(t), \varphi(t), f] d f d \varphi d \theta-S_{0}\right\} d f
$$

\section{Analysis and modelling of the energy-domain characteristics of the electromagnetic environment}

Energy is the basis of electromagnetic activities, and almost all electromagnetic wave applications are based on the propagation of electromagnetic energy. The electromagnetic radiation energy is controlled with various modulation styles in the frequency, time and space domains, which is also the fundamental reason for the mutual influence of various electromagnetic activities. When the power of an electromagnetic interference signal is greater than the operating frequency of an electronic device, interference will occur immediately. The energy-domain characteristic of the electromagnetic environment of a spacecraft reflects the distribution of electromagnetic signal intensity, and its typical performance is the fluctuation of power and the uneven distribution of energy flow. Ideally, electromagnetic radiation propagates in all directions in an infinite space, and the energy density at any point in space is only related to the distance of propagation, whose influencing factor is the attenuation factor. However, in actual situations, due to the existence of various radiation propagation factors, the energy density of electromagnetic radiation in space will not be uniform. The energy-domain characteristics represent the change in electromagnetic signal power, usually expressed by the field intensity, which can reflect the distribution law of signal strength within a specific area, a specific time period and a specific spectrum range ${ }^{29,30}$. According to Equation 4 , in a certain space of $V_{\Omega}$, time range of $\left[\mathrm{t}_{1}, \mathrm{t}_{2}\right]$ and frequency range of $\left[f_{1}, f_{2}\right]$, the signal strength can be expressed as Equation 10, where $S$ expresses the average power density spectrum of the signal.

$$
\mathrm{S}=\frac{1}{V_{\Omega}\left(t_{2}-t_{1}\right)\left(f_{2}-f_{1}\right)} \int_{\Omega} \int_{f_{1}}^{f_{2}} \int_{t_{1}}^{t_{2}} S(\boldsymbol{r}, t, f) d t d f d \Omega
$$

Assuming that the transmitting power of the radiation source is $P_{t}$, the gain of the transmitting antenna in the direction of Device $\mathrm{A}$ is $G_{j r}$, the receiving gain of the receiving antenna of Device A in the direction of the radiation source is $G_{r j}$, and the distance between Device A and the radiation source is R, when only the basic transmission loss $\mathrm{L}$ in free space is considered, the signal power of the radiation source reaching Device A is as follows: 


$$
P_{r}=\frac{P_{t} G_{j r} G_{r j} \lambda^{2}}{(4 \pi R)^{2} L}
$$

Combining the energy-domain discriminant criterion and sensitivity analysis, the energy-domain discriminant criterion calculation model is as follows:

$$
\mathrm{EJ}= \begin{cases}1, & P_{r} \geq P_{r m i n} \\ 0, & P_{r} \leq P_{r m i n}\end{cases}
$$

\section{Conclusion}

During the launch and return of a spacecraft, strong electromagnetic radiation generated by aerospace fuel deflagration and atmospheric friction can interfere with electronic equipment on the spacecraft, blocking the communication connection between the spacecraft and ground and threatens the safety of astronauts. To solve this problem, we first study the characteristics of electromagnetic radiation generated by deflagration. The current known theoretical research suggests that high-energy fuel deflagration causes the surrounding air temperature to rise sharply, forming an unbalanced and incomplete low-temperature plasma region in a certain area, generating electric and magnetic fields that are nonuniformly distributed and changing dynamically. To study the characteristics of electromagnetic radiation, an electromagnetic radiation measurement system composed of antennas, a signal conditioner, remote trigger device and high-speed acquisition card was designed, and an electromagnetic signal analysis method was developed. In the electromagnetic environment modelling section, mathematical models of the electromagnetic environment are constructed based on the spatial, time-domain, frequency-domain and energy-domain characteristics, and the section explores the interference of electromagnetic radiation generated by combustion in the working environment of a spacecraft on electronic equipment, which forms a complete set of "measurement-acquisition-analysis-modelling” analysis projects.

\section{References}

1. Li, W., Zhao, D., Chen, X. et al. Numerical investigation of inlet thermodynamic conditions on solid fuel ramjet performances. International Journal of Aerospace Engineering 2021, 3-4. https://doi.org/10.1155/2021/8868288 (2021).

2. Li, W., Chen, X. \& Qiu, S. Numerical investigation of effect of combustor length on combustion characteristics of solid fuel ramjet. Journal of Physics: Conference Series 1510, 012001. https://doi.org/10.1088/1742-6596/1510/1/012001 (2020).

3. Gao, S., Tian, G., Dai, X. et al. A lightweight wireless qverpressure node based efficient monitoring for shock waves. IEEE/ASME Transactions on Mechatronics 26, 448-457. https://doi.org/10.1109/TMECH.2020.3025955 (2021).

4. Kuhl, A. L., Bell, J. B. \& Beckner, V. E. Heterogeneous continuum model of aluminum particle combustion in explosions. Combustion, Explosion, and Shock Waves 46, 433-448. https://doi.org/10.1007/s10573-010-0058-9 (2010).

5. Kolsky, H. Electromagnetic waves emitted on detonation of Explosives. Nature 173, 77-77. https://www.nature.com/articles/173077a0 (1954).

6. Giri, D. V. \& Tesche, F. M. Classification of intentional electromagnetic environments. IEEE Transactions on Electromagnetic Compatibility 46, 322-328. https://doi.org/10.1109/TEMC.2004.831819 (2004).

7. Adushkin, V. V. \& Soloviev, S. P. Generation of electric and magnetic fields by air, surface, and underground explosions. Combustion, Explosion and Shock Waves 40, 649-657.

https://doi.org/10.1023/B:CESW.0000048266.03133.51 (2004).

8. Boronin, A. P., Vel'Min, V. A., Medvedev, Y. A. et al. Experimental study of the electromagnetic field in the near zone of explosions produced by solid explosives. Journal of Applied Mechanics and Technical Physics 9, 712-717. https://doi.org/10.1007/BF00912873 (1968).

9. Boronin, A. P., Kapinos, V. N., Krenev, S. A. et al. Physical mechanism of electromagnetic field generation during the explosion of condensed explosive charges. Combustion Explosion and Shock Waves 26, 597-602. https://doi.org/10.1007/BF00843137 (1990).

10. Nevels, R. \& Jeong, J. The time domain Green's function and propagator for Maxwell's equations. Antennas \& Propagation IEEE Transactions 52, 3012-3018. https://doi.org/10.1109/TAP.2004.835123 (2004). 
11. Soloviev, S. P. Generation of electric and magnetic field during detonation of high explosive charges in boreholes. Journal of Geophysical Research Solid Earth 110, 1-14. https://doi.org/10.1029/2004JB003223 (2005).

12. Kuhl, A. L., Bell, J. B., Beckner, V. E. et al. Gasdynamic model of turbulent combustion in TNT explosions. Proceedings of the Combustion Institute 33, 2177-2185. https://doi.org/10.1016/j.proci.2010.07.085 (2011). 13. Kuhl, A. L., White, D. A. \& Kirkendall, B. A. Electromagnetic waves from TNT explosions. Journal of Electromagnetic Analysis \& Applications 6, 280-295. https://doi.org/10.4236/jemaa.2014.610028 (2014).

14. Cui, Y. B. \& Kong D. R. Analysis of electromagnetic radiation spectrum during the explosion of energetic materials. 2020 IOP Conf. Ser.: Earth Environ. Sci. 585, 012026. https://doi.org/10.1088/1755-1315/585/1/012026 (2020).

15. Zhu, D. W., Chen, H. L., Chen, B. et al. Improved ADI iterative algorithm with two-step gauss-seidel procedure for efficient laguerre-based BOR-FDTD method. IET Microwaves Antennas \& Propagation 13, 942-949.

https://doi.org/10.1049/iet-map.2018.5653 (2019).

16. Ren, H., Chu, Z. \& Li, J. Study on electromagnetic radiation generated during detonation. Propellants Explosives Pyrotechnics 44, 1541-1553. https://doi.org/10.1002/prep.201900118 (2019).

17. Chen, H., Pan, X., Yong, H. E. et al. Measurement of time-varying electron density of the plasma generated from a small-size cylindrical RDX explosion by Rayleigh microwave scattering. Plasma Science and Technology 23, 045401. https://doi.org/10.1002/prep.201900118 (2021).

18. Jaekel, B. W. Electromagnetic environments - phenomena, classification, compatibility and immunity levels. IEEE EUROCON 2009. 1498-1502. https://doi.org/10.1109/EURCON.2009.5167839 (2009).

19. Gao, S., Tian, G., Dai, X. et al. A novel distributed linear-spatial-array sensing system based on multichannel LPWAN for large-scale blast wave monitoring. IEEE Internet of Things Journal 6, 9679-9688. https://doi.org/10.1109/JIOT.2019.2930472 (2019).

20. Kuhl, A. L., Bell, J. B., Beckner, V. E. et al. Spherical combustion clouds in explosions. Shock Waves 23, 233-249. https://doi.org/10.1007/s00193-012-0410-y (2013).

21. Gao, S., Tian, G. et al. A B-Spline method with AIS optimization for 2-D IoT-Based overpressure reconstruction. IEEE Internet of Things Journal 7, 2005-2013. https://doi.org/10.1109/JIOT.2019.2960827 (2019).

22. Li, C., Chen, X., Li, Y. et al. Role of the backward-facing steps at two struts on mixing and combustion characteristics in a typical strut-based scramjet with hydrogen fuel. International journal of hydrogen energy. 44, 28371-28387. https://doi.org/10.1016/j.ijhydene.2019.09.023 (2019).

23. Musa, O., Chen, X., Li, Y., Li, W. \& Liao, W. H. Unsteady simulation of ignition of turbulent reactive swirling flow of novel design of solid-fuel ramjet motor. Energies 12, 1-32. https://doi.org/10.3390/en12132513 (2019).

24. Zhu, D. W., Wang, Y. G., Chen, H. L. et al. A one-step leapfrog HIE-FDTD method for rotationally symmetric structures. International journal of $R F$ and microwave computer-aided engineering 27, 1-7.

https://doi.org/10.1002/mmce.21081 (2017).

25. Zhu, D .W., Chen, H. L., Yang, J. et al. A novel efficient WLP-based BOR FDTD Method with explicit treating ideology. IEEE Access 7, 16858-16869. https://doi.org/10.1109/ACCESS.2019.2894788 (2019).

26. Xiong, J. L., Wu, Z. C., Sun, Y. \& Liu, X. Y. Analysis and feature modeling of battlefield electromagnetic environment of radio fuze. 2013 Cross Strait Quad-Regional Radio Science and Wireless Technology Conference 2013, $316-320$. https://doi.org/10.1109/CSQRWC.2013.6657418 (2013).

27. Wang, F., Chen, M. \& Wang, L. The discussion on the evaluation method of battlefield electromagnetic environment complexity. 2020 6th International Conference on Big Data and Information Analytics 2020, 440-442. https://doi.org/10.1109/BigDIA51454.2020.00078 (2020).

28. Zhu, S. K., Yang, R. J., Zuo, J. J. \& Li, D. J. Optimization method of OFDM shared signal frequency resource based on complexity of electromagnetic environment. 2019 International Conference on Information Technology and Computer Application (ITCA) 2019, 79-82. https://doi.org/10.1109/ITCA49981.2019.00025 (2019).

29. Tang, X. B., Gao, B. \& Zhang, Y. Design Technology of System-Level EMC Engineering. Berlin, Boston: De Gruyter. https://doi.org/10.1515/9783110562828 (2020). 
30. Peng, H. L. et al. A compact dual-polarized broadband antenna with hybrid beam-forming capabilities. Progress In Electromagnetics Research 118, 253-271. https://doi.org/10.2528/PIER11042905 (2011).

\section{Acknowledgements}

This work was supported by National Natural Science Foundation of China (No. 11372143); in part by the Fundamental Research Funds for the Central Universities under Grant 30919011263; in part by the Natural Science Foundation of Jiangsu Province, China under Grant BK20190464; in part by the Jiangsu Planned Projects for Postdoctoral Research Funds under Grant 1003-YBA20012; in part by the Chinese Postdoctoral Science Foundation under Grant 2020 M671481.

\section{Author contributions statement}

Cui Yuanbo put forward a new idea for thesis topic. Cui Yuanbo and Wang Shuai contributed to design of measurement device and process of analyzing data. Cui Yuanbo and Gao Shang contributed to electromagnetic radiation modeling. Cui Yuanbo wrote the main manuscript text with input from all authors. Kong Deren and Jiang Jian viewed the manuscript and supervised the project.

\section{Competing interests}

The authors declare no competing interests. 